\title{
Review of: "Everybody Nose: Molecular and Clinical Characteristics of Nasal Colonization During Active Methicillin-Resistant Staphylococcus Aureus Bloodstream Infection"
}

\author{
Muhabat Adeola Raji ${ }^{1}$ \\ 1 Alfaisal University \\ Potential competing interests: The author(s) declared that no potential competing interests exist.
}

The manuscript titled "Everybody Nose: Molecular and Clinical Characteristics of Nasal Colonization During Active Methicillin-Resistant Staphylococcus Aureus Bloodstream Infection" reports the characteristics of MRSA isolates from nasal carriers who had concurrent MRSA bloodstream infections.

The study's premise and findings are pertinent to MRSA colonization and invasive disease knowledge gaps.

The study methodology is acceptable. However, I have a question about the processing of the nasal swabs. The authors stated that the "Swabs were plated directly on CHROMID ${ }^{\circledR}$ MRSA plates." I wonder why the authors did not consider vortexing the nasal swabs in nutrient broth or peptone water prior to plating on solid agar. Some studies suggest that bacterial yield is higher than direct swab plating. 